\title{
GLAD!
}

Revue sur le langage, le genre, les sexualités

$05 \mid 2018$

Raconter les sexualités depuis la marge

\section{Baiser la littérature}

Fuck Literature

RER Q collectif d'autriX

\section{(2) OpenEdition}

Journals

Édition électronique

URL : http://journals.openedition.org/glad/1145

ISSN : 2551-0819

Éditeur

Association GSL

Référence électronique

RER Q collectif d'autriX, « Baiser la littérature », GLAD! [En ligne], 05 | 2018, mis en ligne le 15 décembre 2018, consulté le 17 décembre 2020. URL : http://journals.openedition.org/glad/1145

\section{c) (1) $\Theta \Theta$}

La revue GLAD! est mise à disposition selon les termes de la Licence Creative Commons Attribution Pas d'Utilisation Commerciale - Pas de Modification 4.0 International. 

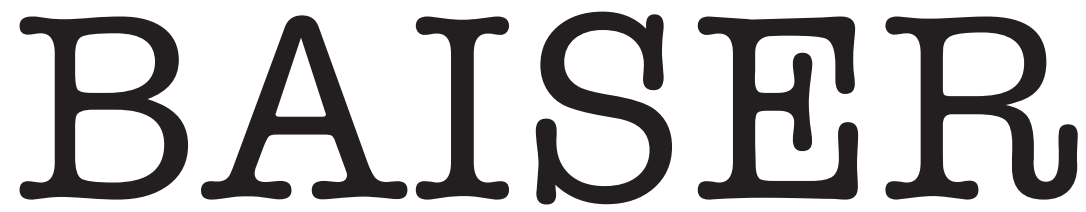

$R E R Q$

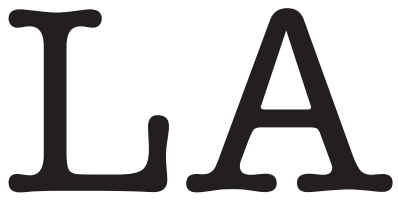

collectif

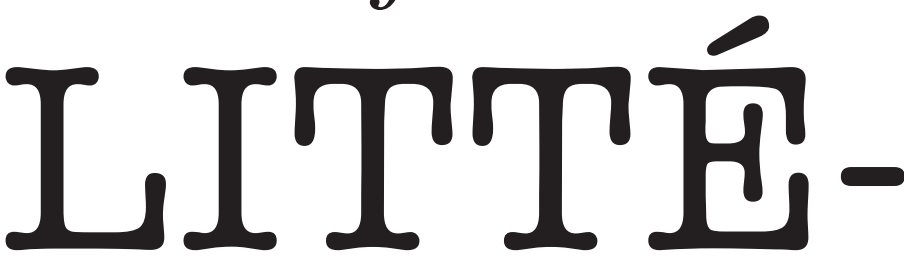

d'autrix

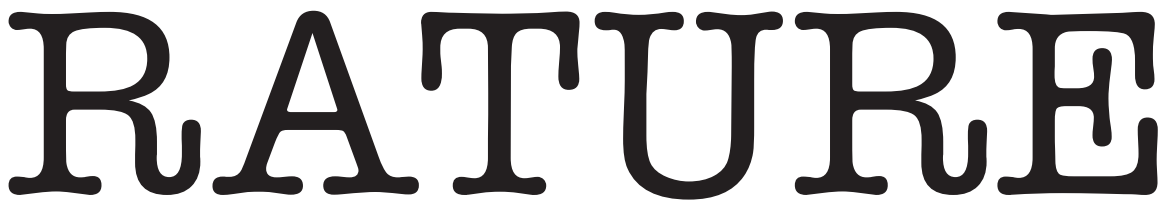

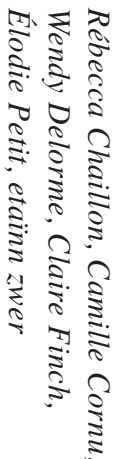




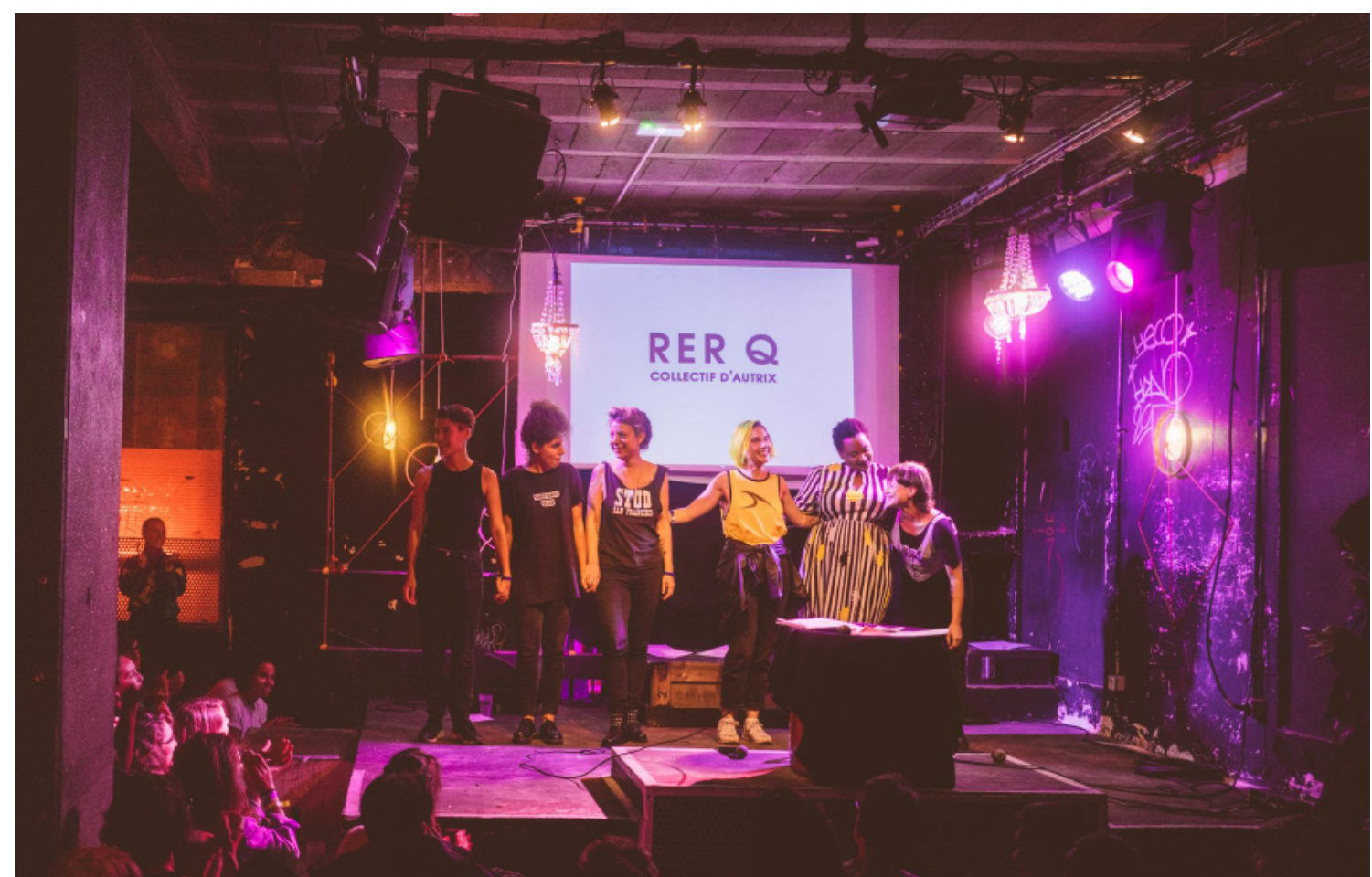

we were born

naked, all the rest is

fiction

RER $\mathbf{Q}$ est un réseau d'autrix alliées autour de textes manifestes queer / crus / cul, RER Q écrit lit performe ce qui n'est que trop rarement visible, RER Q explose le genre triste et la syntaxe molle, la police des corps identifiés identifiables et la littérature officielle, RER $\mathbf{Q}$ serpente station Fulguropoing, arrêt Plug Me Tender - entre les mots d'individues qui racontent leurs perturbations non linéaires dans le genre et la sexualité,
RER Q est substance désir chattes suspectes flemme tantrique trous béantes nuques moites expérimentations sales paysages gouines images clandestines tunnels d'amour fantasmes profonds comme des arbres, RER Q est le voyage néon sans fard sans fin vers ta sexe ta corps ta bouche d'infinie, RER Q est sauvage et multiple et te fait jouir par toutes les interstices 

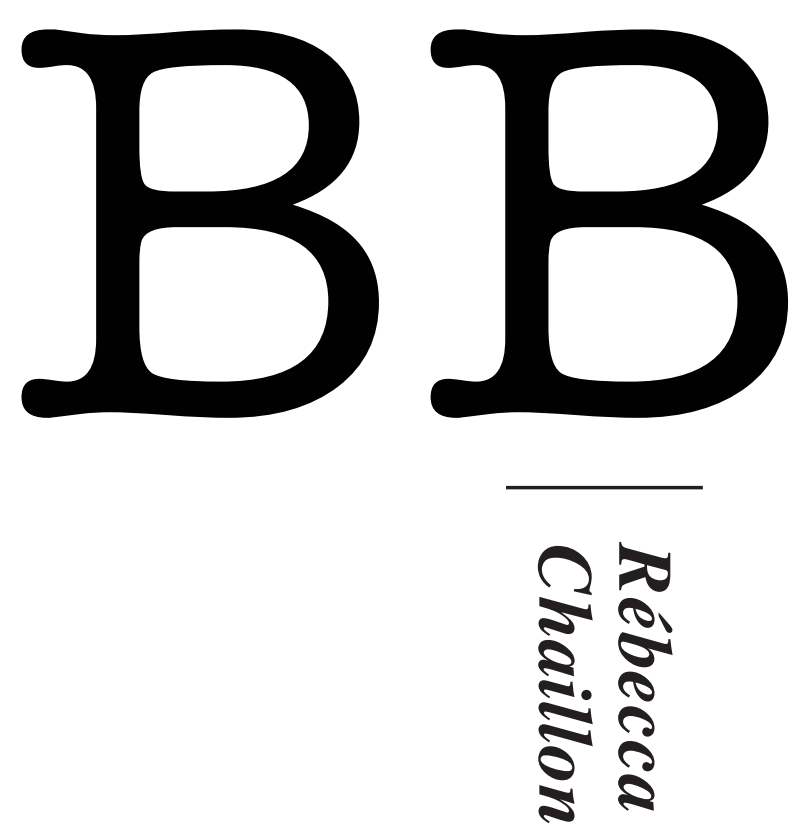

Je repose mon mug de latte aux champignons, je casse mon ampoule détox composée de pissenlit, de pomme, de sureau, de radis noir, et de citron dans une bouteille d'Hépar et je profite du flot pour avaler une gélule d'Harpagophyton. Trente minutes de tuto gainage avec Cindy Crawford et c'est validé. Je me sens saine et sereine pour un barbecue.

Je grossis à vue d'oeil quand je rentre dans ce bar sombre où les corps se révoltent sur de la musique nerveuse, où les murs suintent de mots énervés pour trouver le respect et organiser les luttes. Je n'aime pas avoir ma sexualité écrite sur la gueule depuis que je suis rentrée en payant librement. Mais je ne peux pas m'empêcher de sourire à la vue de crânes rasés abstraitement, de teintures arc-en-ciel délavées, de tétons qui transperçent les marcels, de piercing frais, de genre troublés, de bières pas chère... Je m'en veux que mon instinct de chasseresse vienne ternir ma croyance en l'amour unique.

Ma satiété n'a que des problèmes depuis que je me plais assez pour plaire à autrui. J'essaie d'être poly en restant polie. J'essaie d'assumer que le mot contradiction puisse être tatouable. J'essaie de me mettre en cuisson vapeur. J'essaie.

Je suis excitée rien que par l'odeur du mot.

Les chairs palpitent sous les peaux,

Je les vois s'enflammer

rougir

chauffer et sentir la braise à plein nez.

Surveiller les feux naissants éteindre toute confusion.

Quand je rentre dans ce bar, j'ai l'embarras du choix entre saucisse de poulet et poitrine de porc, cuisses graisseuse, cotelettes osseuses.

je me délecte des corps, qui dansent et frottent, Je rêve de saisir les viandes avec mes doigts, Je rêve de les faire juter dans ma bouche, de laisser couler le sang dans le creux de mes seins de baver, aspirer, me lécher les doigts de saucer

d'y retourner me faire griller, me faire retourner, me brûler.

De ronger jusqu'à la moëlle celle qu'on a oubliée dans un coin. 


\section{distance /}

La sexualité génitale me donne l'impression d'être en cours de biologie au collège

Rien que l'odeur d'un yaourt à la vanille me donne envie de vomir

Et le vomi c'est ma limite

Je ne baise pas en vomissant.

J'ai dépassé le stade génital c'est pas contre ton corps mais les possibilités sont ouvertes et dilatées.

J'ai des ongles, j'ai des dents, j'ai un couteau

J'ai envie de toi

et ta peau est beaucoup trop étanche.

La forme de ton corps ne veut rien dire pour moi Il y a des trous partout là où j'enfonce mes ongles On réinvente nos corps à chaque fois qu'on se touche.

Nous sommes des personnes créatives.

Il ne faut pas dire qu'on aime le sang parce que ça dégoûte les gens, mais je n'ai jamais su me taire au bon moment Pourtant le reste du temps j'y arrive parfaitement.

Le sang dégoûte mes ami.e.s alors je leur donne des pastèques à manger et je les filme

On ne voit pas la même chose à l'écran.

Je traverse une période très fluide rouge mais ça cicatrise vite. 
$\mathrm{X}:$ Tu ne voudrais pas faire du sexe par hasard?

$\mathrm{C}$ : Peut-être mais souvent j'ai juste un peu la flemme.

$\mathrm{X}:$ Tu voudrais faire quoi, cuisiner?

$\mathrm{C}$ : Oui mais après il faudra faire la vaisselle ?

$\mathrm{X}$ : Oui. Tu vois, autant faire du sexe.

$\mathrm{C}$ : Oui mais après il faudra laver tous les godes.

$\mathrm{X}$ : Je pense que depuis le temps qu'on se connaît, c'est bizarre qu'on ne baise pas ensemble.

$\mathrm{C}:$ Ça arrive parfois, de ne pas faire de sexe avec les gens.

$\mathrm{X}$ : C'est une possibilité. Il ne faut pas se priver.

$\mathrm{X}: \mathrm{Si}$ on faisait du sexe tu voudrais (me) faire quoi ?

$\mathrm{C}:$ Te mordre dans une couleur rouge.

$\mathrm{X}$ : Comme dans une pastèque?

$\mathrm{C}:$ Meilleur qu'une pastèque.

$\mathrm{X}: \mathrm{Tu}$ le fais?

$\mathrm{C}:$ Oui.

$\mathrm{X}: \mathrm{Tu}$ le fais?

$C$ : Oui.

$\mathrm{X}:$ Tu le fais?

$\mathrm{C}$ : Oui.

$\mathrm{X}$ : Est-ce qu'on est en train de baiser, là ?

C : J'ai le goût de ton sang dans la bouche, dans les yeux, dans les mains et dans le ventre.

$\mathrm{X}:$ On devrait se lancer.

$\mathrm{C}: \mathrm{Il}$ reste encore beaucoup de possibilités.
$\mathrm{X}$ : Tu me mords dans une couleur rouge, tu t'avances immobile, il y a quelque chose entre nous.

$\mathrm{C}:$ Il y a de la distance entre nous,

$\mathrm{X}:$ Il y a mordre / lécher / griffer / cracher / enculer

$\mathrm{C}:$ Il y a se taire et ne plus y penser

$\mathrm{X}$ : Il y a ma main entière contre les parois de ton vagin

$\mathrm{C}:$ Il y a le jour qui s'est déjà levé on ne l'a même pas remarqué

$X$ : Quelque chose se passe entre nous.

$C$ : Peut-être qu'on est en train de dormir et de rêver.

$\mathrm{X}$ : Je tiens ton corps entier dans le creux de ma main.

$\mathrm{X}:$ Je tiens ton corps entier dans le creux de mes deux mains.

$\mathrm{X}$ : Ma main entière tient dans le creux de ton corps.

$\mathrm{C}: \mathrm{Tu}$ demandes : est-ce que je te fais mal ? Tu dis que je souris.

$\mathrm{X}$ : Tu ne parles pas mais tu souris, je me dis que je peux continuer. Je te retourne, je bloque tes bras derrière ton dos.

$\mathrm{C}$ : J'enlève mes mains, tu les reprends. Tu les remets derrière mon dos. Tu me dis, tu ne bouges pas les bras, ok ? Tu as compris? Tu as compris?

$\mathrm{X}$ : Toi tu ne dis rien.

C : Tu demandes si j'ai compris jusqu'à ce que je dise Oui. Je dis Oui. 
$\mathrm{X}$ : Tu dis oui et je vois tes bras croisés derrière ton dos, ton visage écrasé sur le matelas, tes fesses en hauteur qui viennent vers moi.

$\mathrm{C}$ : Finalement j'enlève mes bras parce que j'ai envie de voir si tu me surveilles. Tu me les remets derrière le dos, tu les tiens d'une main et tu me baises de l'autre. Tu me dis que mon cul doit être plus haut, à la hauteur de ton visage. J'obéis. Tu m'encules en me serrant les bras.

$\mathrm{X}: \mathrm{J}$ 'espère que je ne te fais pas mal. J'espère que toi ça va ? Ça va?

C : J'ai l'impression que tu vas me casser le bras, tu peux desserrer un peu?

$\mathrm{X}$ : Tu aimes juste être maintenue mais dans le confort ? C'est bon à savoir.

$\mathrm{C}:$ Tu me dis : regarde l'effet que tu me fais.

$\mathrm{X}$ : Je suis face à tes hanches énormes, c'est plus grand que tout ce qu'on peut imaginer et pourtant je l'imagine. Regarde l'effet que tu me fais.
J'ai envie de toi

Quand je t'envoie une photo de ma pastèque, tu me dis qu'elle ressemble à un poisson

Tu vis sous l'eau

Tu vis de l'autre côté de la mer.

Tu es étanche.

Je n'imagine aucune griffure aucun trait

Je traverse une période très tranchante mais je ne sais pas faire de choix.

J'ai envie de toi quand tu me dis que toi non plus

J'ai envie de toi et je me dis que je pourrais m'adapter

J'ai envie de toi mais je me dis qu'en fait, non

J'ai envie de toi quand tu n'es pas là puisque tu n'es jamais là

J'ai envie de toi quand j'ai envie de toi

J'ai envie que tu partes et tu restes

J'ai envie que tu reviennes et tu me réponds que tu n'as jamais été là.

J'ai envie de mordre la distance.

J'ai envie de toi quand je rêve

Généralement tu n'es pas là et parfois tu te multiplies tout autour de moi

Il y a des possibilités qui se comptent au-delà de dix doigts Mais généralement tu n'es pas là

Tu vis loin, ça fait beaucoup de kilomètres plus le prix d'un billet d'avion

J'ai envie de toi quand tu me dis que tu ne sais pas ce que c'est qu'un poisson-clown

tu n'as pas vu le monde de Némo

J'ai envie de toi quand même. 


\section{dans la Fluide Mobile \\ les filles sont faites et les désirs floutés}

assises sur un rivage de cuir

leurs cuisses entremêlées leurs bouches érodées (seulement) par le plaisir, je pourrais reconnaître chacune à son rire,

Petite et son tatouage de géantes (qui lui masque tout le dos), La Sombra le feu de sa présence aussi terrible qu'un tableau, Johnnie Johnnie ses mains sèches refermées sur une pierre translucide à force d'être caressée,

et Mercurio, dite vif-argent,

parce que ses yeux

parce que

parce que sa peau couturée d'anneaux

j'ai envie de la lécher, comme un buvard d'acide, comme un timbre sur une lettre d'amour,

c'est elle que j'ai choisie

c'est ce soir, c'est mon tour, c'est comme dans un peep show on roule dans une Cadillac moite

derrière vient une longue traînée de filles perchées sur des vélos cloutés et des skates rose fuchsia

des putes des astrologues des chattes

des tatouées des monstrueuses,

leurs couteaux rangés gentiment

contre leurs sexes chauds

parade au langage inversé

c'est beau et j'ai envie de me jeter dans la nasse, qu'elle me dévore, redevenir minuscule et parcourir chaque cellule sur de lentes pulsations

voilà les Zoo Girls

on traverse la ville dans la nuit qui somnole d'herbes gouines et d'ennui

tout est irradié

c'est ce soir

au club j'avais donné le mot de passe en chuchotant «de quel investissement libidinal suis-je le nom? »

la créature en tuxedo or avait ouvert

et j'avais attendu, saisie, au milieu de corps ambigus et de visages ultra baisables, proie volontaire reflétée à l'infini dans les miroirs salaces

(et) Mercurio m'avait choisie 
c'est ce soir

je me suis préparée, ardemment préparée, ardemment

je me suis branlée (beaucoup), j’ai lu,

je me suis branlée en lisant

des poèmes où il était question : de petites filles (excitées) de

fleurs indéchiffrables de vulves (se mêlant dans l'espace) et

\section{d'utopies}

c'est ce soir

je suis prête j'attends,

leurs gestes en découpe sur ma peau

Mercurio termine son histoire, un conte mythologique

bizarre, bizarrement hypnotique

she spits and travels through the hoops of my veins

her fist rearranges my shape

my self

my will

will $i$ pass the night

the melting the sweet the bloody night

her story keeps me awake

her tongue keeps me awake

her dreams, they're salty

how deep is the sea?,

she asks

and that's how $i$ knew ses mots emplissent l'air de liquides vivaces

dans l'alcôve techno je m'ouvre comme la mer

c'est de la piraterie

il y a un tout petit écart entre ses dents et j'ai envie de glisser ma langue dedans, de m'y nicher toute entière

le silence retombe et il est chargé d'images s'enroulant au ralenti, une pissotière des mirages dans des jockstraps une chambre d'hôtel, des insomnies jaunes comme l'oeil des chats, du velours accidenté

des rêves qui ont l'odeur d'un film passé sous une flamme

alors elle se penche touche ma nuque et je sais :

« couchée »

c'est ce soir, j'obéis,

maintenant

à genoux, bouche ouverte, j'entre dans un récit qui n'a pas encore de nom 
Il y a cet endroit au centre de mon corps, au centre du triangle inversé qui va du bas des côtes jusqu'au nombril. Tu y as plongé tes mains.

Les squelettes n'ont rien à cet endroit-là. Que du vide.

Cela faisait des mois que ce triangle creux était en fusion. Du plomb chaud et liquide.

Tes doigts ont palpé l'arête de mes côtes, ont effleuré la peau, il y avait du tissu car j'étais habillée. Je ne savais pas si tes mains étaient chaudes ou pas. J'avais senti la veille la pulpe de tes doigts, fraîche et précise, sur mon épine dorsale.

J'essaie de mettre en mots ce qui fut du silence et le mouvement des corps.

Une musique jouait, que tu avais choisie, emplissant tout le dôme de tentures que la brise agitait. Par l'ouverture centrale au-dessus de ma tête je regardais le ciel, les branches, une feuille virevoltant, le passage d'un oiseau. Je les chargeais de signes.

J'essaie d'imaginer la suite de cette danse. Le temps était compté, il me fallait partir. J'essaie d'imaginer et ce qui vient à moi ce sont Les Guérillères et c'est Le Corps Lesbien. Dans les pages de Wittig les amantes font des choses qu'on n'imagine pas. Tu pourrais par exemple déshabiller mon corps en enlevant sa peau, comme un simple vêtement. Je n'en souffrirais pas, et tu aurais alors la chair nue et les veines, les tendons, les artères, les organes vivants à portée de ta main. Tu les verrais pulser. Rentrer à l'intérieur n'aurait plus le même sens.
À quoi ressemblerait l'acte de glisser une main dans mon vagin et une langue sur ma vulve, si le derme des muqueuses ne les recouvre plus? Il faudrait que ta peau glisse aussi de ton corps comme un habit serré. On aurait toutes les deux la même consistance, la texture de nos bouches serait pulpe, quartiers de mandarines sorties de leur membrane. Et la lymphe qui suinte de la chair mise à vif ferait glisser nos membres mieux que du lubrifiant. J'imagine l'intense vulnérabilité, l'intensité extrême que prendrait le toucher. Mais cela est possible avec nos épidermes. Tu sais que si tu frottes ta peau à la mienne - j'aurais aimé d'ailleurs sentir sur ma peau nue le contact de la tienne plutôt que du tissu -, il se créera une chose tout à la fois unique et propre à chaque rencontre : l'alchimie de l'odeur de deux peaux qui se chauffent.

Comme tu as su plonger tes mains au centre de mon corps sans entamer la peau, sans entailler les chairs, il me semble possible qu'on puisse se sentir comme des écorchées vives, volontaires et sereines, dépouillées de leur peau et de tous leurs atours - cheveux, poils, rouge à lèvres, cils, gemme portée au cou, boucle d'oreille en plumes - sans n'effectuer aucune entaille ni aucune incision.

Je ne sais pas encore à quoi ressemblerait mon être sans artifices mais je sais qu'on voit bien l'armature du visage sous ta peau sans défaut, et on n'aurait alors pas de genre, plus de nom, on n'aurait rien sur quoi accrocher un fantasme, pas de scénario, nul script déjà connu, 
I hear that most foods were made by accident, repeated mistakes of agitation. The fantasy I have the most frequently now is of licking the base of the double cock, pushing it inside of you. I run my tongue around the opening of your cunt, the thick part of the double cock that's in you, I want to suck your dick. I want to swim underwater. I want to suck somebody's dick underwater wrote David Wojnarowicz. When I translated this I wrote, Je veux nager sous l'eau; sous l'eau je veux sucer la bite de quelqu'un, because French grammar is resistant to repetition, the objects fall out of line when you preserve the original semantic order and besides my friends tell me that the first thing you learn in any composition class it to replace repeated words with synonyms. Subtlety is not at issue but an attestation of ownership of having more words the poverty of a repeated noun or adjective is embarrassing. Replace all words by their synonyms as the heterogeneous text is the good one. All of which means that repetition in French can make you feel serious rhythmic shock and this shock is increased the closer the repeated words are to one another. I want to swim underwater underwater I want to suck someone's dick. I start to fuck you. I'm holding your cock and you're on your back and I'm pulling your cock towards me, I'm pulling up on my hand there the other end of the cock is inside of you and it doesn't make any sound not like that slapping sound you get when you start to fuck someone really hard and your hand or your wrist or your fist around the cock hits skin and slaps. It's deep and it doesn't make any sound until your pussy starts to open up around it, it slips a little, sucking sound of cock moving fast in wet pussy. But what I really want is to

\section{veux}

sucer

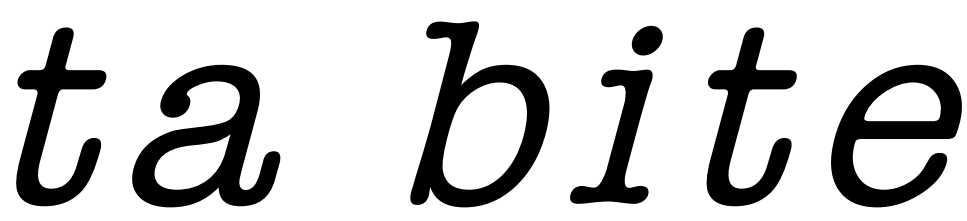

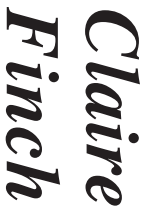

Elle a essayé de traduire cette phrase en français mais ça l'embêtait parce qu'elle pense que la grammaire française refuse les répétitions, il faut prouver qu'on a beaucoup de vocabulaire en utilisant plein de synonymes, pour elle ce refus des répétitions en français est une façon de prouver qu'on possède de l'hétérogénéité. 
Elle commence à baiser son ami, il a la moitié d'un gode dans la chatte et elle a l'autre moitié dans la main, elle le baise comme ça, ça ne fait même pas de bruit, aucun bruit mouillé, et elle a surtout envie de sucer cette bite. Elle essaie de la prendre en entier dans sa bouche et comme ce n'est pas assez mouillé, elle lèche tout autour, elle se l'enfonce dans la bouche jusqu'à la sentir au fond de sa gorge, ça lui donne un haut le coeur, mais elle veut vraiment qu'il lui baise la gorge, elle voudrait moins contrôler la situation, elle se met par terre pendant qu'il reste sur le lit, elle tend sa gorge vers le haut et il s'accroupit audessus d'elle. À ce moment-là, elle se dit que si iels étaient sous l'eau, la gravité lui manquerait.

Elle lui tient les cuisses pour s'enfoncer la bite plus profondément et elle ne peut plus respirer, mais elle prend quand même la peine de bien maintenir la bite au fond de la chatte de son ami pour qu'il continue à lui baiser la bouche, et elle pense encore à la gravité qui lui manquerait si elle était sous l'eau, et l'odeur du lubrifiant mélangé à la salive et à la bave lui manquerait aussi.

Maintenant elle discute au bord d'une piscine gonflable. Quelqu'un lui a dit que sortir du placard n'était pas le comportement le plus professionnel à adopter quand on travaille dans l'art contemporain, mais elle se demande si on peut vraiment mettre de côté nos particularités, ou si chaque décision esthétique ne serait pas conditionnée par l'expérience ? Le féminisme est partout, et le sexe est partout. suck your dick. I crawl on the floor push your body push my hands on your stomach to hold you down, immediately try to take the whole cock into my mouth. It's not wet enough so I lick around it. This time I move my lips down it, feel the tip of your cock at the back of my throat and gag, I want you to fuck my throat. I have too much control here I need you to do it for me I need you to push my hands off of you, roll over stand over me where I am on the floor my head against some furniture, my legs are on the floor but my throat is coming and it's searching for your cock, you crouch over me. Underwater I think I would miss what gravity is doing here. I can't breathe. I'm holding your thighs just under your ass, pulling your cock closer. With one hand I push on the part of the cock that's coming out of your cunt, I hold it in you, I'm holding you as you're fucking my mouth, underwater we would miss how gravity makes us feel, like, I think we would miss the smell of lube from earlier mixed with silicone and spit.We're sitting in beach chairs next to an inflatable pool and a performer who does this thing with sounds is wearing earplugs because she's sensitive to noise. The earplugs are the cheap orange kind you get on airplanes or in bathrooms at clubs so she can't hear anything, she leans in close like she's going to whisper something then she shouts directly in my ear. "My work isn't political," she says, "My artistic commitment is so much bigger than politics, like it's outside of politics. Because I'm a professional artist no one knows I'm a lesbian. I don't want my work to be reduced to a stigma, I've seen how that goes." Of course stigma is real and especially in France anyone over the age of 40 will tell you that the best professional decision you can make is to stay closeted but, I want to ask her, can transcendence overcome the limits of our particularity, or is every aesthetic decision inflected by experience? EVERYTHING IS FEMINISM AND EVERYTHING IS SEX. 


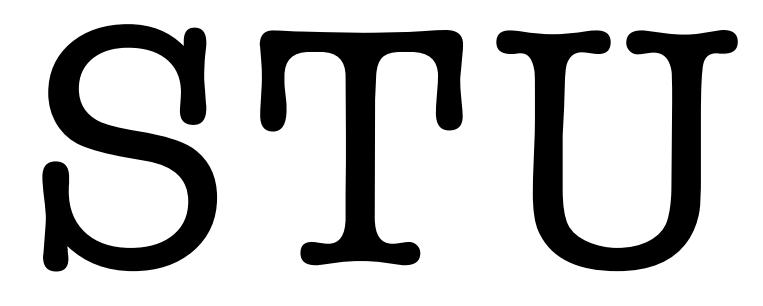

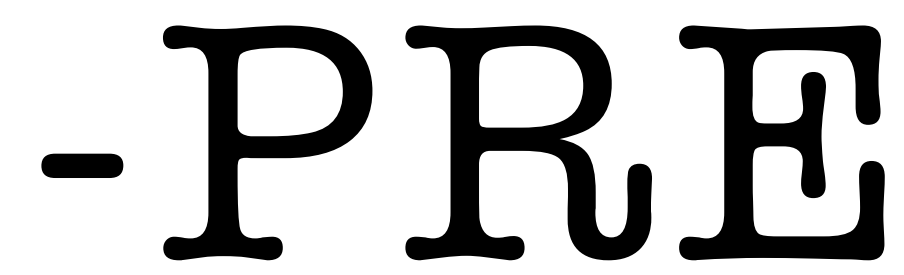

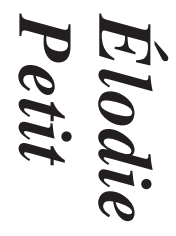

\section{Arthur \\ Rimbaud}

la gouine je m'appelle arthur rimbaud et je suis gouine

une vraie gouine qui aime l'autre trempée aux bouts de mes doigts serrés ma peaux qui colle aux leurs ma chatte qui suffoque leurs cuisses

j' aime garder ma veste quand je baise j' aime quand ça répond direct en face de moi

j'imagine serrer ta nuque très fort pendant que tu me suces ma veste toujours sur mes épaules mon poing à l'entrée de ton cul tu t'ouvres trempée et impuissante tu ris

arthur rimbaud 
je ne suis pas alcoolique

j'ai peur de la mort

le sexe à la vie m'attache

c'est une histoire de fluide

je ne veux pas de chamane à dix mille balles

je ne veux pas léguer mon existence au travail salarial

je crois en la séduction permanente

à comment tu t'habilles pour que je te déshabille

j'espère qu'il fera chaud et que ton cul sera salé que chacune se mettra à niquer puissamment et fort

arthur rimbaud mon avenir proche est une rentrée sombre entourée de personnes intellectuelles, faussement bourgeoises ou faussement pauvres

on performe la précarité et l'intelligence artificielle

tout sera critiqué et réduit à néant

le langage épuisé et ma chatte dans ton nez

il ne restera plus rien

alors

je mets mes bas mes bas sont mis le nylon de mes bas dans le ciel étoilé sur ton sexe écrasé le goudron mouillé

arthur rimbaud

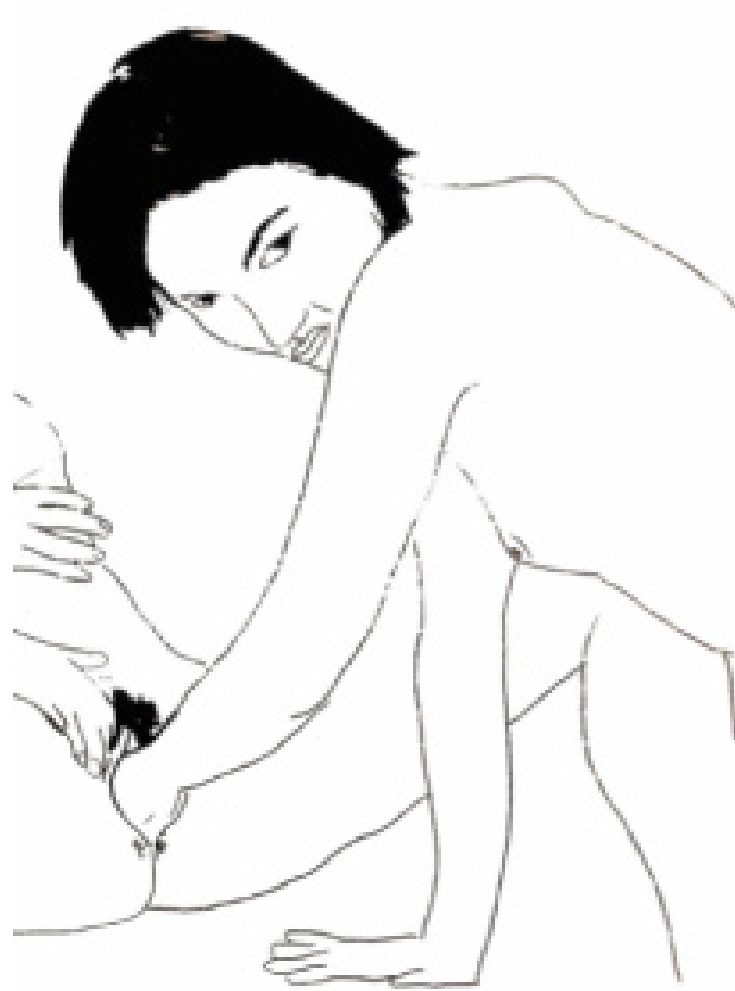




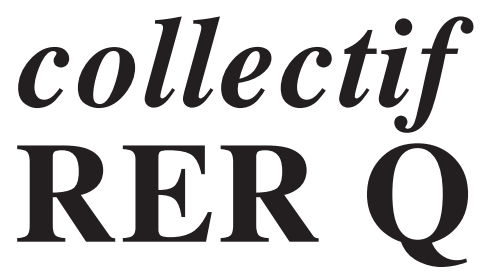

